\title{
Ueber die Umwandlung der Stärke im thierischen Organismus.
}

\author{
Von \\ Dr. E. H. Bimmermamn \\ aus Amsterdam.
}

Vor einiger Zeit veröffentlichten v. Mering und Musculus in der Zeitschrift für physiologische Chemie (Bd. II) einen Artikel: Ueber die Umwandlung von Stärke und Glycogen durch Speichel, Pancreas- und Leberferment, worin sie ihre Entdeckung mittheilten, dass durch Einwirkung genannter Fermente auf Amylum und Glycogen Maltose in bedeutender Menge, Traubenzucker in Spuren auftrete.

Ferner bringen sie neue Beweismittel für die Hinfälligkeit der Seegen'schen Behauptung, laut der es je nach der Nahrung verschiedene Modificationen von Glycogen geben sollte. Bereits auf der 49. Naturforscherversammlung hielt v. Mering ${ }^{1}$ ) einen Vortrag uiber Diabetes mellitus, in welchem er unter Anderem anführte, dass er das Glycogen, welches nach Zufuhr von Kohlenhydraten als auch dasjenige, welches nach $\mathrm{Zufuhr}$ von Albuminaten entsteht, gegen Fermente etc. geprüft habe, „ohne, dass sich hierbei ein Unterschied ergab". Dieser Angabe geben nun v. Mering und Musculus noch mehr Beweiskraft, da es ihnen gelang, aus Glycogen, welches nach Fibrinfütterung und ans Glycogen, welches nach Amylaceennahrung auftritt, dieselben Zersetzungsproducte (reducirendes Achroodextrin, Maltose und Traubenzucker) nachzuweisen. - Vor wenigen Monaten hat Musculus ${ }^{2}$ ) im Vereine mit Gruber von neuem gezeigt, dass Stärke durch Diastase oder Säuren eine mit Wasseraufnahme verbundene Spaltung erfährt.

1) v. Mering: Untersuchungen über Diabetes mellitus. 'Tageblatt der 49. Naturforscherversammlung in Hamburg. Ref. in No. 40 der deutschen Zeitschrift für pract. Medizin 1876.

2) Zeitschrift für phys. Chemie. Bd. II. 
Die Körper, welche hierbei gebildet werden, sind lösliche Stärke, Dextrine, Maltose und Traubenzucker. Die verschiedenen Dextrine nennen sie, je nachdem sie leichter oder schwerer durch Fermente angegriffen werden, Achroodextrin $\alpha$, Achroodextrin $\beta$ und Achroodextrin $\gamma$ : Seegen $\mathbf{n}^{\mathbf{1}}$ ) fand später für letzteres den wohlklingenden Namen Dystropodextrin.

Es lag nun nahe zu untersuchen, wie sich diese verschiedenen Spaltungsprodukte der Stärke im thierischen Organismus verhalten würden. Zunächst wollte ich aber die Angaben von Musculus und v. Mering in Bezug auf die Umwandlung der Stärke durch thierische Fermente näher prüfen. $\mathrm{Zu}$ diesem Behuf liess ich Speichel auf Stärke einwirken und konnte ich hierbei, wie dies folgender Versuch zeigt, Maltose und Spuren von Traubenzucker nachweisen.

$100 \mathrm{gr}$ Kartoffelstärke wurden mit $1000 \mathrm{ccm}$ Wasser verkleistert und mit $800 \mathrm{ccm}$ filtrirtem Mundspeichel versetzt. Nachdem die Flüssigkeit 5 Stunden' lang bei $30-40^{\circ}$ erwärmt worden war, wurde sie auf dem Wasserbade bis zum Syrup eingedampft und mit $1400 \mathrm{ccm}(95 \%)$ Alkohol versetzt. Zum alkoholischen Filtrat wurden $1000 \mathrm{ccm}$ Aether gefügt; es bildete sich hierdurch ein Niederschlag, von dem nach zwei Tagen sich die darüber stehende Flüssigkeit klar trennen liess. Zum Filtrat wurden nun zwei Liter Aether gesetzt. - Nach 36 Stunden hatte sich ein reichlicher, krystallinischer Niederschlag gebildet. Die Krystalle wurden einer näheren Prüfung unterzogen und erwiesen sich als reine Maltose.

Durch fortgesetztes Fällen mit Aether gelang es schliesslich in der ätherisch-alkoholischen Lösung geringe Mengen von Traubenzucker nachzuweisen.

Aus Glycogen, welches nach Traubenzuckerfütterung und aus Glycogen, welches, nach Eiweissfütterung entstanden war, konnte ich durch Einwirkung von Speichel und Pancreassaft grössere Mengen von Maltose, sowie Tranbenzucker ḍarstellen. Ferner erhielt ich hierbei ein reducirendes Dextrin.

Was nun meine Versuche betrifft, so will ich, um später nicht in W iederholungen zu fallen, kurz die Methode meiner Untersuchung angeben. Als Versuchsthiere wurden Kaninchen benutzt und die be-

1) Pflüger's Archiv 1879. Bd. XIX. 
treffenden Körper (durchschnittlich $2 \mathrm{gr}: 30 \mathrm{cem}$ Wasser) in eine Jugularvene langsam und möglichst gleichmässig injicirt. Das vierundzwanzigstïndige Urinquantum wurde sorgfältig gesammelt und durch Thierkohle filtrirt; dadurch ging zwar ein geringes Quantum Zucker verloren, es war mir jedoch nicht darum zu thun quantitative, sondern qualitative Zuckerbestimmungen im Harn zu machen.

Im Anfange benutzte ich zur Titrirung des Harns Fehling'sche Lösung, musste dieselbe jedoch bald verlassen, weil in vielen Fällen keine Endreaction auftrat, sondern statt ihrer eine schmutzig griun bräunliche Färbung des Harns, welche nicht mehr verschwand. ' Statt der Fehling'schen Flitssigkeit benutzte ich eine Quecksilberlösung, welche Sachse angegeben hat:

$18 \mathrm{gr}$ reines und trockenes Jodquecksilber werden mit Hïlfe von $35 \mathrm{gr}$ Jodkali in Wasser gelöst. Za dieser lı̈sung fügt, man 80. gr Aetzkali in Wasser gelöst und verdünnt das Ganze auf $1000 \mathrm{ccm} .40 \mathrm{ccm}$ Quecksilberlösung =0,72 gr Jodquecksilber entsprechen 0,15 Traubenzucker. Die Ausfühung der Bestimmung geschieht in der Weise, dass man eine gewisse Menge z. B. $40 \mathrm{ccm}$ der Qnecksilberlösung in einer Porcellanschale zum Sieden bringt und die zuckerhaltige Flüssigkeit aus einer Bürette zufliessen lässt, bis alles Quecksilber ausgefällt ist. Bei Zusatz der Zuckerlösung zu der heissen Quecksilberlösung wird die Mischung sofort trïbe, klärt sich aber am Ende der Operation und wird etwas gelblich. Zur Erkennung der Endreaction wendet Sachsse eine alkalische Zinnoxydullösung an, welche man sich durch Uebersättigung einer Lösung des käuflichen Zinnsalzes mit Alkali bereitet. Eine solche Lösung fällt Quecksilber augenblicklich und erzeugt auch noch in sehr verdünnten Lösungen dieses Metalles eine deutliche braune Trübung. Man bringt einige Tropfen der Zinnoxydullösung in kleine Porcellanschälchen und setzt hierzu von Zeit zu Zeit einige Tropfen der Quecksilberlösung. Anfangs entsteht ein starker schwarzer Niederschlag, der um so geringer wird, je mehr die Fällung des Quecksilbers durch den Traubenzucker fortschreitet; sabald auch die Bräunung beim Vermischen beider Flüssigkeiten verschwunden ist, ist alles Quecksilber ausgefällt, der Versuch also beendigt. Man kann recht gut anf diese Weise nach Sachsse noch den Erfolg von $1 / 10 \mathrm{ecm}$ einer halbprocentigen Zuckerlösung $=0,0005$ Zucker beobachten. Ich kann diese vorzuigliche Methode, besonders bei zoochemischen Untersuchungen, wo wir es häufig 
mit stark gefärbten zuckerhaltigen Flüsșigkeiten zu thun haben und die Fehling'sche Lösung keine scharfe Endreaction erkennen lässt, nur empfehlen.

Zuerst untersuchte ich den ursprünglichen durch Kohle filtrirten Urin, später jedoch dampfte ich den Harn zur Syrupconsistenz ein und extrahirte dann den Syrup mit Alcohol, dampfte denselben $a b$ und löste den Rückstand in Wasser. Hierdurch kann man Maltose und Traubenzucker zum grossen Theil von den Dextrinen trennen, was in verschiedenen Versuchen von Wichtigkeit war. Die Lösung wird in den Versuchsprotokollen alcoholischer Extract genannt. Dasjenige, was sich. in Alcohol nicht löste, wird in Wasser gelöst und wässeriger Extract genańnt. Beide Extracte wurden dann in Bezug auf Drehungsund Reductionsvermögen sowie mitunter auf Gährungsfähigkeit untersucht. Der ursprïngliche Urin wurde auch stets auf Albumen gepriuft.

\section{Versuchsprotocolle.}

\section{Maltose.}

Spec. Drehungsvermögen $\alpha]=+150$, Spec. Reductionsvermögen $=66$.

Versuch 1.

Einem mittelgrossen Kaninchen werden 2 gr Maltose injicirt.

$98 \mathrm{ccm}$ Urin werden aufgefangen.

Derselbe enthält nach der

$$
\begin{aligned}
& \text { Pol. }=1,33 \% \text { Traubenzucker } \\
& \text { Red. }=0,45 \%
\end{aligned}
$$

oder nach der

$$
\begin{aligned}
& \text { Pol. }=0,44 \% \text { Maltose } \\
& \text { Red. }=0,68 \%
\end{aligned}
$$

$60 \mathrm{ccm}$ werden zur Syrupconsistenz eingedampft und mit absolutem Alcohol anfgenommen.

Der alcohol. Extract enthielt nach der

$$
\begin{aligned}
& \text { Pol. }=0,253 \% \text { Traubenzucker, } \\
& \text { Red. }=0,225 \%
\end{aligned}
$$

oder nach der

$$
\begin{aligned}
& \text { Pol. }=0,09 \% \text { Maltose } \\
& \text { Red. }=0,34 \%
\end{aligned}
$$




\section{Versuch 2.}

Einem grossen Kaninchen werden 2 gr Maltose injicirt. $200 \mathrm{ccm}$ Urin aufgefangen.

Nach der Pol. würde er 0,699\% Traubenzucker,

enthalten.

Red. " " $0,373 \%$

oder nach der Pol. würde er 0,274\% Maltose

und " "Red. " " $0,559 \%$ "

enthalten.

Nach Kochen mit verdünnter Schwefelsäure nahm das Reductionsvermögen des Urin zu und das Drehungsvermogen ab; mithin enthielt er Maltose. Ungefähr $100 \mathrm{ccm}$ werden eingedampft mit absol. Alkohol aufgenommen und mit ein wenig Aether versetzt, die ätherisch-alkoholische Lösung enthält nach der

$$
\begin{aligned}
& \text { Pol. }=0,21 \% \text { Traubenzucker, } \\
& \text { Red. }=0,22 \%
\end{aligned}
$$

Nach Kochen mit verdünnter Salzsäure sieht man keine Zunahme der Reduction eintreten; also haben wir es mit reiner Glycose zu thun.

\section{Versuch 3.}

Einem grossen Kaninchen werden $2 \mathrm{gr}$ Maltose injicirt.

$120 \mathrm{ccm}$ Urin aufgefangen.

Der Alcohol-Extract $(95 \%)$ enthält nach der

Pol. $=1,19 \%$ Glycose oder $0,396 \%$ Maltose,

Red. $=0,585 \% \quad$ " $\quad 0,887 \% \quad$ "

Diese Versuche ergeben, dass Maltose im Blute theilweise in Traubenzucker umgewandelt, theilweise unverändert den Organismus verlässt.

\section{Lösliche Stärke.}

Spec. Rotationsvermögen $\alpha]=+218$. Spec. Reductionsvermögen $=6$.

\section{Versuch 1.}

Einem Kaninchen werden 2,5 gr. lösl. Stärke injicirt. Urinquantum $120 \mathrm{ccm}$.

Der Harn zeigt keine Jodreaction. Derselbe drehte, wie wenn er 2,48\% Glycose enthielt und reducirte, als enthielte er $0,75 \%$ Glycose. Auf Zusatz 
von Speichel nahm das Reductionsvermögen des Urins um 13\% zu, das Drehungsvermögen wesentlich $\mathrm{ab}$. Der, alkoholische Extract des ursprünglichen Urins gährte mit Hefe deutlich. Der Rest des Urins wurde nach Vergährung untersucht; er drehte, als enthielte er 1,5\% Glycose und reducirte, als enthielte er $0,25 \%$ Glycose.

\section{Versuch 2.}

Einem grossen Kaninchen werden $3 \mathrm{gr}$ lösliche Stärke injicirt. Nach 4 Stunden werden $48 \mathrm{~cm}$ Urin ausgepresst. Derselbe zeigt keine Jodreaction, dreht, als enthielte er 6,69\% Glycose und reducirt, als enthielte er 1,56 Glycose.

Ein Theil des Urins wird mit $20 \mathrm{ccm}$ Speichel 3 Stunden stehen gelassen. Das Reductionsvermögen des Urins nahm hierdurch um stark 10 Procent zu. Nach Kochen mit verdünnter Schwefelsäure zeigte der Urin ein zweifach höheres Reductionsvermögen.

In der 2. Probe Urin, welche nach 16 Stunden aufgefangen wurde und $95 \mathrm{ccm}$ betrug, fand sich nach der Reduction $0,26 \%$ Glycose und nach der Polarisation 0,25\% Glycose. Auf Zusatz von Hefe trat deutliche Gährung ein. Durch Kochen mit verdünnter Schwefelsäure wurde das Reductionsvermögen nicht gesteigert.

Aus diesen Versuchen geht hervor, dass lösliche Stärke im Blute amgewandelt wird, da der Urin gegen Jodlösung ein negatives Verhalten zeigt. Als Zersetzungsproducte der löslichen Stärke lassen sich mit Sicherheit im Urin Traubenzucker sowie Dextrin nachweisen. Der Nachweis von Dextrin stützt sich sowohl auf die Zunahme des Reductionsvermögens, welche durch Zusatz von Speichel anftrat, als auch auf die sehr beträchtliche Steigerung des Reductionsvermögens, welche nach Kochen mit verdiunnter Sehwefelsäure zu constatiren war. $\mathrm{Ob}$ auch Maltose, welche von Speichel in mehreren Stunden nicht angegriffen wird, im Urin vorhanden ist, lässt sich mit Sicherheit aus diesen Versuchen nicht entscheiden, dünkt uns aber sehr wahrscheinlich, da bei der Saccharification des Amylums sich hauptsäehlich Maltose bildet und Maltose zum Theil den Organismus unverändert passirt, wie wir oben gezeigt haben. 


\section{Achroodextrin $\alpha$.}

Spec. Rotationsvermögen $\alpha]=+210$, Spec. Reductionsvermögen $=12$.

\section{Versuch 6.}

Einem kleinen Kaninchen werden 2 gr Achroodextrin $\boldsymbol{*}$ injicirt. $160 \mathrm{ccm}$ Urin werden aufgefangen.

Der Urin dreht, als enthielte er $3,03 \%$ Glycose und reducirt, als enthielte er $1,04 \%$ Glycose.

Ungefähr $100 \mathrm{ccm}$ Harn werden bis zur Syrupconsistenz eingedampft und mit $(70 \%)$ Alkohol ausgezogen. Alkoholischer Extract dreht, als enthielte er 2,89\% Glycose und reducirt, als enthielte er $0,7 \%$ Glycose. Der alkoholische Extract gährt deutlich.

Der alkoholische Extract wird mit dem Rest des Urins mit Hefe versetzt und nach beendeter Gährung untersucht. Die Lösung drehte nach Vergährung, als enthielte sie 0,79\% Glycose und reducirte, als enthielte sie $0,1 \%$ Glycose. Nach Kochen mit verdünnter Schwefelsäure stieg das Reductionsvermögen beträchtlich.

\section{Versuch 7.}

Einem grossen Kaninchen werden 2,5 gr Achroodextrin $\alpha$ injicirt.

$150 \mathrm{ccm}$ Urin aufgefangen, zur Syrupconsistenz eingedampft and mit Alkohol $(95 \%)$ aufgenommen. Alkoholischer Extract enthält nach der Polarisation 3,52\% Glycose und nach der Reduction 1,19\% Glycose. Nach Vergährung des alkoholischen Extractes dreht die Lösung, als enthielte sie 0,84\% Traubenzucker und reducirt, als enthielte sie $0,11 \%$ Traubenzucker. Durch Kochen mit verdünnter Säure nimmt das Reductionsvermögen wesentlich zu.

\section{Versuch 8.}

Einem kleinen Kaninchen werden 2 gr Achroodextrin $\alpha$ injicirt.

$80 \mathrm{ccm}$ Urin werden aufgefangen.

Der alkoholische Extract drehte, als enthielte er 2,0\% Glycose und reducirte, als enthielte er $0,8 \%$ Glycose.

Der alkoholische Extract vergährte mit Hefe vollends, so dass nach Vergährung in der Lösung nur ein minimales Reductionsvermögen zu constatiren war.

Der wässerige Extract drehte, als enthielte er 0,79\% Glycose und reducirte; als enthielte er $0,12 \%$ Glycose. Auf Zusatz von Hefe trat keine Gährung ein. 
Aus diesen drei Versuchen geht hervor, das s Achroodextrin $\alpha$ im Blute nur eine theilweise Umwandlung erleidet. Als Zersetzungsproducte finden sich Traubenzucker und Maltose. Dextrin blieb nach Vergährung des Urins itbrig und wurde als solches durch Drehung, Reduction etc. erkannt.

\section{Achroodextrin $\beta$.}

Spec. Rotationsvermögen $\alpha]=+190$, spec. Reductionsvermögen 12 .

\section{Versuch 9.}

Einem Kaninchen werden 2,5 gr Achroodextrin $\beta$ injicirt.

$125 \mathrm{ccm}$ Urin aufgefangen.

Der gesammte Harn wird zur Syrupoonsistenz eingedampft und mit Alcohol $(70 \%)$ aufgenommen.

Der alkohol. Extract dreht, als enthielte er $0,76 \%$ Glycose und reducirt, als enthielte er $0,3 \%$ Glycose.

Derselbe gährt mit Bierhefe ein wenig. Der wässerige Extract dreht, als enthielte er $3,11 \%$ Glycose und reducirt, als enthielte or $0,41 \%$ Glycose; mit $\mathrm{HCl}$ versetzt und im Kochsalzbade während drei Stunden gekocht, findet man nach der

$$
\begin{aligned}
& \text { Pol. }=0,96 \% \text { Glycose } \\
& \text { Red. }=1,05 \%
\end{aligned}
$$

\section{Versuch 10.}

Einem grossen Kaninchen werden 2,5 gr Achroodextrin $\beta$ injicirt.

$160 \mathrm{ccm}$ Urin aufgefangen.

Dieselben werden bis zur Syrupconsistenz eingedampft und mit absolutem Alcohol ausgezogen.

Der alcoholische Extract enthält nach der

$$
\begin{aligned}
& \text { Pol. }=0,27 \% \text { Glycose, } \\
& \text { Red. }=0,28 \%,
\end{aligned}
$$

Der wässerige Extract dreht, als enthielte or $7,3 \%$ Glycose und reducirt, als enthielte er 1,1\% Glycose.

Nach Kochen mit $\mathrm{HCl}$ im Salzbade findet man nach der

$$
\begin{aligned}
& \text { Pol. }=1,64 \% \text { Glycose, } \\
& \text { Red. }=1,76 \%
\end{aligned}
$$

Aus diesen beiden Versuchen ergibt sich, dass Achroodextrin $\beta$ zum Theil in Zucker und zwar in Traubenzucker umgewandelt wird; ob auch Maltose gleichzeitig vor- 
handen, lässt sich nicht bestimmt sagen, erscheint uns jedoch wahrscheinlich. Ferner findet sich im Urin-Dextrin.

\section{Achroodextrin $\gamma$.}

Spec. Rotationsvermögen $\alpha]=+150$, spec. Reductionsvermögen 18 .

Versuch 11 .

Einem grossen Kaninchen werden 2 gr Achroodextrin $\gamma$ injicirt. 4 Stunden post $58 \mathrm{ccm}$ Urin aufgefangen.

Derselbe dreht, als enthielte er $1,93 \%$ Glycose und reducirt wie $0,61 \%$ Glycose.

Diese Probe Urin wird mit dem noch später aufgefangenen $(40 \mathrm{ccm})$ zusammen eingedampft und mit $70 \%$ Alcohol aufgenommen. Der alcoholische Extract gährt nicht.

\section{Versuch 12.}

Einem grossen Kaninchen werden $21 / 2$ gr Achroodextrin $\gamma$ injicirt.

$125 \mathrm{ccm}$ Urin aufgefangen.

Der Alcoholextract $(95 \%)$ dreht, als enthielte er $0,94 \%$ Glycose und reducirt, als enthielte er $0,21 \%$ Glycose.

Derselbe wird mit ausgewaschener Hefe versetzt; er gährt nicht. Hierauf wird er filtrirt und mit verdünnter Salzsäure während 3 Stunden im Salzbade gekocht. Er enthält nun nach der

$$
\begin{aligned}
& \text { Pol. }=0,45 \% \text { Glycose. } \\
& \text { Red. }=0,48 \%
\end{aligned}
$$

Der wässerige Extract enthält nach der Pol. $=3,29 \%$ Glycose, und nach der Red. $=0,40 \%$ Glycose. Nach Kochen mit verdünnter Salzsäure im Salzbade enthält er nach der Polarisation $=1,01 \%$ Glycose und nach der Reduction $=1,24 \%$ Glycose.

Diese beiden Versuche zeigen, dass nach intravenöser Injection von Achroodextrin $y$ in Urin keinZucker auftritt, da im alkoholischen Extracte keine gährungsfähige Substanz vorhanden war. Vielleicht wird das Dextrin $\gamma$ im Blute in ein stärker reducirendes Dextrin nmgewandelt.

So sehen wir, dass Stärke im Blute, -- wenn auch leichter, - so doch in derselben Weise umgewandelt wird, als dies durch thierische und pflanzliche diastatische Fermente ausserhalb des Organismus geschieht.

So z. B. gibt lösliche Stärke und Dextrin $\alpha$ leicht gährungsfähigen Zucker, Dextrin $\beta$ sehwer und Dextrin $\gamma$ gar keinen, wie 
dies auch in Gegenwart von Fermenten geschieht. Maltose hingegen, welche durch Fermente nicht angegriffen wird, erleidet im Blutstrom zwar eine geringe, aber doch deutliche Umwandlung in Traubenzucker. Die Zunahme des Reductionsvermögens, welche Dextrin $\gamma$ erleidet, ohne dass sich Zucker bildet, dentet auf die Bildung eines neuen von Dextrin $\gamma$ verschiedenen Dextrin. Ein solehes Dextrin, welches schon von mehreren Autoren unter dem Namen Dextrin $\delta$ angegeben, scheint wirklich anfutreten, wenn Amylum mit stärkeren Mitteln angegriffen wird als mit Fermenten oder mit verdünnten Säuren.

(Aus dem physiologischen Laboratorium von Prof. Ad. Dr. C. Ustimowitsch in St. Petersburg.)

\section{Zur Lehre über die Innervation der Blutbahn.}

Von

Joh. Pawlow.

(Vorläufige Mittheilung.)

1) Durchschneidung der Halsvagi beim Hunde.

Im Jahre 1869 (the Journal of Anat. and Physiol. Maiheft) ist eine kurze Mittheilung von Prof. Rutherford, die Wirkung der Vagusdurchschneidung auf den Blutdruck betreffend, veröffentlicht worden.

Rutherford's Erfahrungen gemäss hängt der Erfolg der Vagusdurchschneidung vom Zustande des Verdauungskanals ab: bei vollem Magendarmkanal soll nämlich die Nervendurchschneidung eine Drucksteigerung auslösen, wogegen an nüchternen Thieren diese Operation keine Blutdrucksehwankungen bewirke.

Den Grund dieser Erscheinung will Rutherford auf eine depressorische Gefässerweiterung der Baucheingeweidegefässe durch die Nahrung zurïckführen. Es sollen nämlich die im Vagusstamm verlaufenden sensiblen (depressorischen) Bahnen von der Darmwand aus durch die Nahrung gereizt werden. Die Trennung dieser 